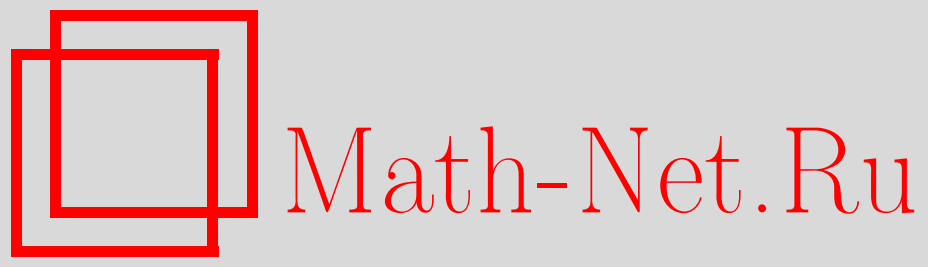

В. Ю. Королев, И. Г. Шевцова, О точности нормальной аппроксимации. II, Теория вероятн. и ее примен., 2005, том 50, выпуск 3, 555-564

DOI: https://doi.org/10.4213/tvp95

Использование Общероссийского математического портала MathNet.Ru подразумевает, что вы прочитали и согласны с пользовательским соглашением

http://www.mathnet.ru/rus/agreement

Параметры загрузки:

IP : 54.162 .27 .143

26 апреля 2023 г., 13:00:04

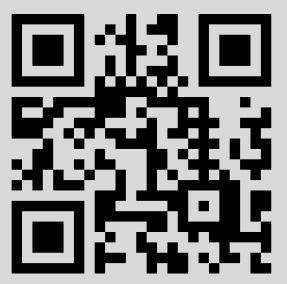


2. Запорожец Д. Н. Случайные полиномы и геометрическая вероятность. -- Докл. PAH, 2005, т. 400, № 3, с. 299-303.

3. Ибрагимов И. А., Маслова Н.Б. О среднем числе вещественных нулей случайных полиномов. I. Коэффициенты с нулевым средним. - Теория вероятн. и ее примен., 1971, т. 16, в. 2 , с. $229-248$.

4. Ибрагимов И.А., Маслова Н.Б. О среднем числе вещественных нулей случайных полиномов. II. Коэффициенты с ненулевым средним. - Теория вероятн. и ее примен., 1971, т. 16 , в. 3, с. 495-503.

5. Ибрагимов И.А., Маслова Н.Б. Среднее число вещественных корней случайных полиномов. - Докл. АН СССР, 1971, т. 199, № 1, с. 13-16.

6. Ибрагимов И. А., Подкорытов С. С. О случайных вещественных алгебраических поверхностях. - Докл. РАН, 1995, т. 343, № 6, с. 734-736.

7. Шпаро Д. И., Шур М. Г. О распределении корней случайных многочленов. - Вест. Моск. ун-та, 1962 , № 3, с. 40-43.

8. Bloch A., Pólya $G$. On the roots of certain algebraic equations. - Proc. London Math. Soc., 1931, v. 33, p. 102-114.

9. Edelman A., Kostlan E. How many zeros of a random polynomial are real? - Bull. Amer. Math. Soc., 1995, v. 32, № 1, p. 1-37.

10. Hammersley J.M. The zeroes of a random polynomial. - Proceedings of the Third Berkeley Symposium on Mathematical Statistics and Probability. V. II. Berkeley-Los Angeles: Univ. California Press, 1956, p. 89-111.

11. Ibragimov I., Zeitouni $O$. On roots of random polynomials. - Trans. Amer. Math. Soc., 1997 , v. 349, №6, p. 2427-2441.

12. Kac $M$. On the average number of real roots of a random algebraic equation. - Bull. Amer. Math. Soc., 1943, v. 49, p. 314-320.

13. Kac $M$. On the average number of real roots of a random algebraic equation. II. Proc. London Math. Soc., 1949, v. 50, p. 390-408.

14. Littlewood J.E., Offord A.C. On the number of real roots of a random algebraic equation. I. - J. London Math. Soc., 1938, v. 13, p. 288-295.

15. Littlewood J.E., Offord A.C. On the number of real roots of a random algebraic equation. II. - Proc. Cambridge Philos. Soc., 1939, v. 35, p. 133-148.

16. Littlewood J.E., Offord A.C. On the number of real roots of a random algebraic equation. III. - Матем. сб., 1943, т. 12, № 3, с. 277-286.

17. Logan B.F., Shepp L. A. Real zeros of random polynomials. - Proc. London Math. Soc., 1968, v. 18, p. 29-35.

18. Logan B.F., Shepp L. A. Real zeros of random polynomials. II. - Proc. London Math. Soc., 1968 , v. 18 , p. 308-314.

19. Shepp L.A., Vanderbei R. J. The complex zeros of random polynomials. - Trans. Amer. Math. Soc., 1995, v. 347, № 11, p. 4365-4384.

Поступила в редакцию 12.IV.2005

(C) $2005 \Gamma$.

КОРОЛЕВ В. Ю. , ШЕВЦОВА И. Г.*

\section{О ТОЧНОСТИ НОРМАЛЬНОЙ АППРОКСИМАЦИИ. II $^{1)}$}

Приведены оценки асимптотически правильных констант в оценках точности нормальной аппроксимации для распределений сумм независимых одинаково распределенных случайных величин с конечными момен-

* Московский государственный университет им. М.В. Ломоносова, факультет вычислительной математики и кибернетики, Ленинские горы, 119992 Москва, Россия; e-mail: vkorolev@comtv.ru

1) Работа выполнена при поддержке Российского фонда фундаментальных исследований (проекты 03-01-00428, 04-01-00671, 05-07-90103). 
тами порядка $2+\delta, 0<\delta<1$. Построены уточненные практически применимые оценки точности нормальной аппроксимации, при этом правая часть оценки представляет собой сумму двух слагаемых, первое из которых являе'гся ляпуновской дробью с абсолютной константой, близкой к асимптотически правильной, а второе убывает быстрее $n^{-\delta / 2}$. При этом для второго слагаемого получены явные оценки и специальные «разложения».

Ключевые слова и фразы: центральная предельная теорема, нормальная аппроксимация, неравенство Берри-Эссеена, оценка скорости сходимости, асимптотически правильная константа.

1. Введение. Пусть $X_{1}, X_{2}, \ldots$ - независимые одинаково распределенные случайные величины, удовлетворяющие условиям

$$
\mathbf{E} X_{1}=0, \quad \mathbf{D} X_{1}=\sigma^{2}<\infty .
$$

Функцию распределения случайной величины $X_{1}$ обозначим $F(x)$, ее характеристическую функцию - $f(t)$. Введем аналогичные обозначения для функции распределения и характеристической функции нормированной суммы $S_{n}=\left(X_{1}+\cdots+X_{n}\right) /(\sigma \sqrt{n})$ :

$$
F_{n}(x)=F^{* n}(x \sigma \sqrt{n}), \quad f_{n}(t)=\left[f\left(\frac{t}{\sigma \sqrt{n}}\right)\right]^{n} .
$$

Функцию распределения и плотность стандартного нормального закона обозначим $\Phi(x)$ и $\varphi(x)$ соответственно:

$$
\Phi(x)=\frac{1}{\sqrt{2 \pi}} \int_{-\infty}^{x} e^{-t^{2} / 2} d t, \quad \varphi(x)=\frac{1}{\sqrt{2 \pi}} \exp \left\{-\frac{x^{2}}{2}\right\} .
$$

Центральная предельная теорема утверждает, что последовательность функций распределения нормированных сумм $S_{n}$ случайных величин, удовлетворяющих условию (1), при $n \rightarrow \infty$ равномерно сходится к стандартной нормальной функции распределения:

$$
\rho\left(F_{n}, \Phi\right) \equiv \sup _{x}\left|F_{n}(x)-\Phi(x)\right| \longrightarrow 0
$$

Известно, что при условии существования абсолютного момента порядка $2+\delta$ с $0<\delta \leqslant 1$, т.е. при условии

$$
\beta^{2+\delta} \equiv \mathbf{E}\left|X_{1}\right|^{2+\delta}<\infty
$$

величина $\rho\left(F_{n}, \Phi\right)$ имеет порядок $n^{-\delta / 2}$. А именно, справедливо неравенство

$$
\rho\left(F_{n}, \Phi\right) \leqslant C_{\delta} L_{n}^{2+\delta}
$$

где

$$
L_{n}^{2+\delta}=\frac{\beta^{2+\delta}}{\sigma^{2+\delta} n^{\delta / 2}}
$$

а $C_{\delta}$ - положительная абсолютная постоянная (см., например, [8, гл. VI, теорема 6]). При $\delta=0$ нормальная сходимость имеет место, но может быть как угодно медленной $[6]$.

Для случая $\delta=1$ наилучшая на сегодняшний день верхняя оценка соответствующей константы $C_{1}$ в неравенстве (3) равна 0.7655 [11].

Для случая $0<\delta<1$ в работе [16] (см. также [15]) получена следующая таблица значений оценок константы $C_{\delta}$ :

\begin{tabular}{|c|c|c|c|c|c|c|c|c|c|}
\hline$\delta=$ & 0.1 & 0.2 & 0.3 & 0.4 & 0.5 & 0.6 & 0.7 & 0.8 & 0.9 \\
\hline$C_{\delta} \leqslant$ & 1.102 & 1.076 & 1.008 & 0.950 & 0.902 & 0.863 & 0.833 & 0.812 & 0.802 \\
\hline
\end{tabular}


Л. Падитц [14] показал, что при $\delta=0$ имеет место неравенство

$$
\rho\left(F_{n}, \Phi\right) \leqslant 3.51 \cdot \frac{1}{\sigma^{2}} \mathbf{E}\left(X_{1}^{2} \min \left\{1, \frac{\left|X_{1}\right|}{\sigma \sqrt{n}}\right\}\right)
$$

откуда вытекает, что имеет место равномерная по $\delta \in[0,1)$ оценка $C_{\delta} \leqslant 3.51$, так как при любом $\delta \in(0,1]$ выражение в правой части последнего неравенства не превосходит $3.51 \cdot L_{n}^{2+\delta}$.

Для случая $\delta=1$ известны многие работы (см., например, [13], [2], [12], [10]), в которых разрыв между точностью, устанавливаемой неравенством (3), и реальной точностью нормальной аппроксимации компенсируется за счет отыскания минимально возможной константы $\underline{C}$, обеспечивающей выполнение неравенства

$$
\rho\left(F_{n}, \Phi\right) \leqslant \underline{C} L_{n}^{3}+o\left(L_{n}^{3}\right) .
$$

Еще в работе [13] было показано, что $\underline{C}=(\sqrt{10}+3) /(6 \sqrt{2 \pi}) \approx 0.4098$ (дальнейшие усилия были направлены на поиск оптимальной оценки для $O\left(L_{n}^{3}\right)$, см. исторический обзор и соответствующий список литературы в [4]).

В то же время для случая $0<\delta<1$ практически ничего не известно об оценках наименьших возможных констант $\underline{C}(\delta)$, обеспечивающих при любых распределениях $F$, удовлетворяющих условиям (1) и (2), справедливость неравенства

$$
\rho\left(F_{n}, \Phi\right) \leqslant \underline{C}(\delta) L_{n}^{2+\delta}+o\left(L_{n}^{2+\delta}\right) .
$$

Другими словами,

$$
\underline{C}(\delta)=\sup \limsup _{n \rightarrow \infty}\left(L_{n}^{2+\delta}\right)^{-1} \rho\left(F_{n}, \Phi\right),
$$

где супремум берется по всем распределениям $F$, удовлетворяюшим условиям (1) и (2). Данная статья посвящена проблеме оценивания асимптотически правильных констант $\underline{C}(\delta)$ для случая $0<\delta<1$ и, как следствие, построению уточненных практически вычислимых оценок точности нормальной аппроксимации для распределений сумм независимых одинаково распределенных случайных величин. Эта задача, наряду с теоретическим, представляет некоторый интерес и с точки зрения практических приложений, в которых используется нормальная аппроксимащия (например, в теории надежности, теории управления запасами, актуарной и финансовой математике), поскольку в большинстве практических задач объем выборки $n$ фиксирован. Вопрос о точности нормальной аппроксимации для распределений сумм слагаемых, распределения которых имеют тяжелые (в некотором смысле) хвосты, приобретает дополнительную важность в связи с необходиостью решать задачи, связанные с большими рисками. Случай $0<\delta<1$ как раз соответствует ситуации, в которой слагаемые имеют настолько тяжелые хвосты, что у них отсутствуют моменты третьего порядка.

В работе [4] мы рассмотрели «гладкий» случай, когда распределения слагаемых имеют ограниченную плотность. Здесь мы рассматриваем случай произвольных распределений слагаемых. Мы выписываем практически вычислимую оценку для $o\left(L_{n}^{2+\delta}\right)$ в $(4)$ и оцениваем асимптотически правильную константу $\underline{C}(\delta)$. Мы приводим функцию $C(\delta)$ такую, что $\underline{C}(\delta) \leqslant C(\delta)$, причем в зависимости от $\delta$

$$
(0.0665 \approx) \frac{1}{6 \sqrt{2 \pi}} \leqslant C(\delta) \leqslant \frac{1}{\pi} \quad(\approx 0.3184)
$$

(левая граница соответствует значениям $\delta$, близким к единице, правая - значениям $\delta$, близким к нулю).

В книге [9, с. 611] отмечено, что «в последнее время большое внимание уделялось обобшениям теоремы Берри-Эссеена на величины, не имеюшие момента третьего порядка. В этом случае граница в неравенстве выражается через момент дробного порядка или какую-нибудь родственную величину.... Обычные вычисления в этом случае достаточно запутаны, а попьтки разработать универсальнье методы, применимье ко многим случаям, не предпринимались.... Необходимость в моментах 
третьего порядка появляется в доказательстве теоремы [Берри-Эссеена] только из-за неравенства

$$
\left|e^{i t x}-1-i t x+\frac{(t x)^{2}}{2}\right| \leqslant \frac{|t x|^{3}}{6} \gg .
$$

В большинстве работ, посвященных изучению ситуации, когда существуют лишь моменты порядка $2+\delta$, неравенство (5) используется в некотором конечном интервале изменения аргумента $x$, а для остальных значений аргумента используется граница порядка $(t x)^{2}$ (см., например, [7], [8], [1]). Для доказательства наших результатов (см. лемму 1 ниже) вместо упомянутого метода усечения, основанного на использовании неравенства (5), мы используем иной подход, базирующийся на оценке

$$
\left|e^{i x}-\left(1+i x+\cdots+\frac{(i x)^{n}}{n !}\right)\right| \leqslant \frac{2^{1-\delta} \Gamma(1+\delta)|x|^{n+\delta}}{\Gamma(n+1+\delta)}, \quad x \in \mathbf{R}
$$

справедливой для любого целого $n \geqslant 0$ и для любого $\delta \in(0,1]$. Это позволяет использовать традиционную универсальную схему рассуждений, основанную на применении классического неравенства сглаживания и его модификаций, ориентированных на оптимизацию абсолютных констант. Доказательство неравенства (6) (в неявной форме) можно найти, например, в [5, с. 212-213].

Здесь и далее символ $\Gamma(\cdot)$, как обычно, обозначает эйлерову гамма-функцию,

$$
\Gamma(y)=\int_{0}^{\infty} z^{y-1} e^{-z} d z, \quad y>0 .
$$

Всюду далее для упрошения записей, не ограничивая обшности, мы полагаем $\sigma^{2}=1$.

2. Вспомогательные результаты. Пусть $d-$ некоторое число, лежашее в интервале $(0, \sqrt{2})$. Введем функции

$$
\begin{aligned}
& a_{1}(d)=\frac{1}{4} \sum_{r=2}^{\infty} \frac{1}{r}\left(\frac{d^{2}}{2}\right)^{r-2}=-\frac{1}{d^{4}}\left[\frac{d^{2}}{2}+\ln \left(1-\frac{d^{2}}{2}\right)\right], \\
& a_{2}(d)=d^{2-\delta} a_{1}(d)+\frac{2^{1-\delta}}{(1+\delta)(2+\delta)}, \\
& b_{1}(d)=\frac{1}{2}-d^{\delta / 2} a_{2}(d) .
\end{aligned}
$$

Несложно видеть, что

причем

$$
a_{1}(d)=\frac{1}{4} \sum_{r=2}^{\infty} \frac{1}{r}\left(\frac{d^{2}}{2}\right)^{r-2}=\frac{1}{4}\left[\frac{1}{2}+\sum_{r=3}^{\infty} \frac{1}{r}\left(\frac{d^{2}}{2}\right)^{r-2}\right]
$$

так что

$$
\sum_{r=3}^{\infty} \frac{1}{r}\left(\frac{d^{2}}{2}\right)^{r-2}=\sum_{j=1}^{\infty} \frac{1}{j+2}\left(\frac{d^{2}}{2}\right)^{j} \leqslant \frac{1}{3} \sum_{j=1}^{\infty}\left(\frac{d^{2}}{2}\right)^{j}=\frac{d^{2}}{3\left(2-d^{2}\right)}
$$

и, стало эыть,

$$
\lim _{d \rightarrow 0+} a_{1}(d)=\frac{1}{8}
$$

$$
\lim _{d \rightarrow 0+} a_{2}(d)=\frac{2^{1-\delta}}{(1+\delta)(2+\delta)}, \quad \lim _{d \rightarrow 0+} b_{1}(d)=\frac{1}{2} .
$$

Более того, можно убедиться, что функция $b_{1}(d)$ монотонно убывает при $d \in(0, \sqrt{2})$, причем на интервале $(0, \sqrt{2})$ лежит единственный нуль этой функции, который мы обозначим $d_{0}(\delta)$.

Лемма 1. Пусть выполнены условия (1) $и$ (2) при $0<\delta \leqslant 1$. Тогда для любого $d \in(0, \sqrt{2})$ и любого $n \geqslant 1 n p u|t| \leqslant d \sqrt{n} / \beta^{2+\delta}$ справедлива оченка

$$
\left|f_{n}(t)-e^{-t^{2} / 2}\right| \leqslant a_{2}(d) \frac{\beta^{2+\delta}}{n^{\delta / 2}}|t|^{2+\delta} \exp \left\{-b_{1}(d) t^{2}\right\} .
$$


Доказательство, основанное на применении неравенства (6), см. в [4].

Пусть $v>0$ и $\gamma>0$ - произвольные числа. Рассмотрим плотность вероятностей

$$
p_{v, \gamma}(x)=\frac{\gamma}{2 \pi v}\left(\frac{\sin (\gamma x /(2 v))}{\gamma x /(2 v)}\right)^{2}, \quad x \in \mathbf{R} .
$$

Несложно убедиться, что плотности $p_{v, \gamma}(x)$ соответствует характеристическая функция

$$
g_{v, \gamma}(t)=\left\{\begin{array}{lll}
1-\frac{v}{\gamma}|t|, & \text { если } & |t| \leqslant \frac{\gamma}{v} \\
0, & \text { если } & |t|>\frac{\gamma}{v}
\end{array}\right.
$$

Обозначим

$$
\alpha=\alpha(\gamma)=\int_{-v}^{v} p_{v, \gamma}(x) d x \quad\left(=\frac{2}{\pi} \int_{0}^{\gamma / 2}\left(\frac{\sin y}{y}\right)^{2} d y=\frac{2}{\pi \gamma}(\gamma \operatorname{Si}(\gamma)+\cos \gamma-1)\right) .
$$

Пусть $\gamma_{0}$ - решение уравнения

$$
\alpha(\gamma)=\frac{1}{2}
$$

Численные расчеты показывают, что $\gamma_{0} \approx 1.69957$. Для всех $\gamma>\gamma_{0}$ имеем $\alpha(\gamma)>\frac{1}{2}$.

Лемма 2. Для любьх $\gamma>\gamma_{0} u v>0$ справедливо неравєнство

$$
\rho\left(F_{n}, \Phi\right) \leqslant \frac{1}{2 \alpha(\gamma)-1}\left[\frac{1}{2 \pi} \int_{-\gamma / v}^{\gamma / v}\left|\frac{f_{n}(t)-e^{-t^{2} / 2}}{t}\right|\left|g_{v, \gamma}(t)\right| d t+\frac{v \alpha(\gamma)}{\sqrt{2 \pi}}\right] .
$$

Доказательство леммы дословно повторяет доказательство леммы 12.2 из [1].

\section{3. Основные результаты.}

Теорема 1. Пусть выполнены условия (1) $и$ (2) для некоторого $0<\delta \leqslant 1$. Тогда для любого $n \geqslant 1$ справедливо неравенство

$$
\rho\left(F_{n}, \Phi\right) \leqslant C_{n}(\delta) L_{n}^{2+\delta}
$$

гde

$$
\begin{aligned}
C_{n}(\delta) & =\inf _{\substack{\gamma_{0}<\gamma<\infty \\
0<d<d_{0}(\delta)}}\left\{\frac{1}{2 \alpha(\gamma)-1}\left[K_{\delta}(d)+\frac{\gamma \alpha(\gamma)}{\sqrt{2 \pi} d n^{(1-\delta) / 2}}\right]\right\}, \\
K_{\delta}(d) & =\frac{\Gamma((2+\delta) / 2) a_{2}(d)}{2 \pi\left[b_{1}(d)\right]^{1+\delta / 2}} .
\end{aligned}
$$

Поскольку $C_{n}(\delta)$ - невозрастающая функция $n$ при каждом $\delta \in(0,1]$, справедливо следующее утверждение.

Следствие 1. В условиях теоремы 1 неравенство (3) справедливо с $C_{\delta} \leqslant C_{1}(\delta)$, zde

$$
C_{1}(\delta)=\frac{1}{\sqrt{2 \pi}} \inf _{\substack{\gamma=<<\infty \\ 0<d<d_{0}(\delta)}}\left\{\frac{1}{2 \alpha(\gamma)-1}\left[\frac{\Gamma((2+\delta) / 2) a_{2}(d)}{\sqrt{2 \pi}\left[b_{1}(d)\right]^{1+\delta / 2}}+\frac{\gamma \alpha(\gamma)}{d}\right]\right\} .
$$

Значения константы $C_{1}(\delta)$ при некоторых $\delta$ приведены в таблице.

\begin{tabular}{||c|c||c|c||c|c||}
\hline$\delta$ & $C_{1}(\delta)$ & $\delta$ & $C_{1}(\delta)$ & $\delta$ & $C_{1}(\delta)$ \\
\hline 0.05 & $9.7036 \cdot 10^{7}$ & 0.40 & 6.1990 & 0.75 & 3.2426 \\
\hline 0.10 & $6.8100 \cdot 10^{4}$ & 0.45 & 5.2118 & 0.80 & 3.1219 \\
\hline 0.15 & 365.8883 & 0.50 & 4.5772 & 0.85 & 3.0229 \\
\hline 0.20 & 54.9891 & 0.55 & 4.1387 & 0.90 & 2.9408 \\
\hline 0.25 & 20.2670 & 0.60 & 3.8199 & 0.95 & 2.8721 \\
\hline 0.30 & 11.3497 & 0.65 & 3.5790 & 1.00 & 2.8143 \\
\hline 0.35 & 7.9034 & 0.70 & 3.3916 & & \\
\hline
\end{tabular}


Несмотря на то, что приведенные значения оценки абсолютной константы $C_{1}(\delta)$ в целом довольно невелики, они все же заметно хуже значений, полученных в рабо$\operatorname{тax}[16]$ и [14]. Возможно, оценки абсолютной константы $C_{1}(\delta)$ могут быть уточнены за счет использования более тонкого неравенства сглаживания, нежели то, которое составляет утверждение леммы 2. Тем не менее, теорема 1 позволяет получить вполне приемлемые и, по крайней мере для некоторых диапазонов $\delta$, неулучшаемые оценки асимптотически правильной константы $\underline{C}(\delta)$ в $(4)$. Этому и посвяшена оставшаяся часть данного пункта.

Учитывая соотношения (10), легко видеть, что на интервале $\left(0, d_{0}(\delta)\right)$ функция $K_{\delta}(d)$ монотонно и непрерывно возрастает, причем

$$
\lim _{d \rightarrow 0+} K_{\delta}(d)=\frac{2^{1-\delta / 2} \Gamma((\delta+2) / 2)}{\pi(1+\delta)(2+\delta)} \equiv C(\delta), \quad \lim _{d \rightarrow d_{0}(\delta)-} K_{\delta}(d)=+\infty .
$$

Поэтому для любого $\varepsilon>0$ существует корень $d(\delta, \varepsilon)$ уравнения

$$
K_{\delta}(d)=C(\delta)+\varepsilon,
$$

лежащий в полуинтервале $\left(0, d_{0}(\delta)\right)$. При этом $d(\delta, \varepsilon)$ как функция $\varepsilon$ монотонно и непрерывно возрастает от $0+$ до $d_{0}(\delta)$ при $\varepsilon$, изменяюшемся от $0+$ до $+\infty$.

Легко видеть, что при $\gamma$, изменяюшемся от $\gamma_{0}$ до $\infty$, величина $\alpha(\gamma)$ монотонно и непрерывно возрастает от $\frac{1}{2}$ до 1 . Поэтому на указанном интервале изменения параметра $\gamma$ функция $w(\gamma)=(2 \alpha(\gamma)-1)^{-1}$ монотонно и непрерывно убывает от $+\infty$ до 1. Стало быть, для любого $\omega>0$ существует конечный корень $\gamma(\omega)$ уравнения

$$
w(\gamma)=1+\omega,
$$

причем $\gamma(\omega)$ монотонно и непрерывно убывает от $+\infty$ до $\gamma_{0}$ при $\omega$, изменяюшемся от 0 до $+\infty$. При этом

$$
\alpha(\gamma(\omega))=\frac{2+\omega}{2(1+\omega)} .
$$

Принимая во внимание сказанное, из теоремы 1 с учетом (14) мы получаем следующий результат, который делает более наглядным вид коэффициента $C_{n}(\delta)$.

Следствие 2. В условиях (1) $и$ (2) при $0<\delta \leqslant 1$ для любого $n \geqslant 1$

$$
\rho\left(F_{n}, \Phi\right) \leqslant \inf _{\varepsilon>0, \omega>0}\left\{(C(\delta)+\varepsilon)(1+\omega)+\frac{(2+\omega) \gamma(\omega)}{2 \sqrt{2 \pi} d(\delta, \varepsilon) n^{(1-\delta) / 2}}\right\} \frac{\beta^{2+\delta}}{n^{\delta / 2}} .
$$

Пусть $\left\{\omega_{n}\right\}_{n \geqslant 1}$ и $\left\{\varepsilon_{n}\right\}_{n \geqslant 1}$ - две последовательности положительных чисел такие, что

$$
\omega_{n} \downarrow 0 \quad \text { и } \quad \varepsilon_{n} \downarrow 0
$$

при $n \rightarrow \infty$. Выберем эти последовательности так, чтобы

$$
\frac{\gamma\left(\omega_{n}\right)}{d\left(\delta, \varepsilon_{n}\right)}=o\left(n^{(1-\delta) / 2}\right) \quad \text { при } \quad n \rightarrow \infty
$$

(такой выбор возможен в силу монотонной и непрерывной зависимости функций $\gamma(\omega)$ и $d(\delta, \varepsilon)$ от своих аргументов, обоснованной выше). Тогда, подставляя указанные $\omega_{n}$ и $\varepsilon_{n}$ в вытекающее из следствия 2 неравенство

$$
\rho\left(F_{n}, \Phi\right) \leqslant(C(\delta)+\varepsilon)(1+\omega) \frac{\beta^{2+\delta}}{n^{\delta / 2}}+\frac{(2+\omega) \gamma(\omega)}{2 \sqrt{2 \pi} d(\delta, \varepsilon)} \frac{\beta^{2+\delta}}{\sqrt{n}},
$$

мы приходим к заключению о справедливости следуюшего утверждения.

Следствие 3. Пусть выполнень условия (1) и (2) при $0<\delta<1$. Тогда неравенство (4) справедливо при $\underline{C}(\delta) \leqslant C(\delta)$, где $C(\delta)$ определено в (11), причем для величины о $\left(L_{n}^{2+\delta}\right)$ в правой части неравенства (4) справедливо представление

$$
o\left(L_{n}^{2+\delta}\right)=\left(\varepsilon_{n}+\omega_{n} C(\delta)+\varepsilon_{n} \omega_{n}\right) \frac{\beta^{2+\delta}}{n^{\delta / 2}}+\frac{\left(2+\omega_{n}\right) \gamma\left(\omega_{n}\right)}{2 \sqrt{2 \pi} d\left(\delta, \varepsilon_{n}\right)} \frac{\beta^{2+\delta}}{\sqrt{n}} .
$$


Другими словами, в условиях (1) и (2) при $\delta \in(0,1)$

$$
\underline{C}(\delta) \leqslant \inf _{n} C_{n}(\delta)=\lim _{n \rightarrow \infty} C_{n}(\delta)=C(\delta) \equiv \frac{2^{1-\delta / 2} \Gamma((2+\delta) / 2)}{\pi(1+\delta)(2+\delta)} .
$$

Значения константы $C(\delta)$ при некоторых $\delta$ приведены в таблице.

\begin{tabular}{||c|c||c|c||c|c||}
\hline$\delta$ & $C(\delta)$ & $\delta$ & $C(\delta)$ & $\delta$ & $C(\delta)$ \\
\hline 0.05 & 0.2867 & 0.40 & 0.1515 & 0.75 & 0.0907 \\
\hline 0.10 & 0.2592 & 0.45 & 0.1399 & 0.80 & 0.0850 \\
\hline 0.15 & 0.2352 & 0.50 & 0.1294 & 0.85 & 0.0797 \\
\hline 0.20 & 0.2141 & 0.55 & 0.1201 & 0.90 & 0.0750 \\
\hline 0.25 & 0.1955 & 0.60 & 0.1116 & 0.95 & 0.0706 \\
\hline 0.30 & 0.1791 & 0.65 & 0.1040 & 1.00 & 0.0665 \\
\hline 0.35 & 0.1645 & 0.70 & 0.0970 & & \\
\hline
\end{tabular}

Сопоставляя эту таблицу с таблицей из работы [16], мы замечаем, что при всех значениях $\delta \in(0,1)$ константа $C(\delta)$ сушественно меньше константы $C_{\delta}$. При этом отношение $C_{\delta} / C(\delta)$ изменяется от 4 (при малых $\delta$ ) до примерно 11 (при $\delta$, близких к единице). Минимальное же значение отношения $C_{1}(\delta) / C(\delta)$ превосходит 35 .

Несложно убедиться, что

$$
\sup _{0<\delta \leqslant 1} C(\delta)=\lim _{\delta \rightarrow 0+} \frac{2^{1-\delta / 2} \Gamma((2+\delta) / 2)}{\pi(1+\delta)(2+\delta)}=\frac{1}{\pi}<0.31831 .
$$

Поэтому мы получаем следуюшую равномерную по $\delta$ оценку асимптотически правильной абсолютной константы $\underline{C}(\delta)$.

Следствие 4. В условиях (1) $u(2)$ с $\delta \in(0,1)$ выполнено неравенство $\underline{C}(\delta) \leqslant 1 / \pi$.

3 а м е ч а н и е 1 . Асимптотически правильная константа $\underline{C}(\delta)$ не является непрерывной функцией аргумента $\delta$ при $0<\delta \leqslant 1$, так как она имеет разрыв в точке $\delta=1$ : из следствия 3 вытекает, что $\lim _{\delta \rightarrow 1-} \underline{C}(\delta) \leqslant \lim _{\delta \rightarrow 1-} C(\delta)=1 /(6 \sqrt{2 \pi})$, более того, из асимптотического разложения Эссеена вытекает, что в случае $\delta=1$ число $1 /(6 \sqrt{2 \pi})$ является неулучшаемой, асимптотически правильной константой для случая нерешетчатых распределений слагаемых. В то же время, как мы уже отмечали, в работе [13] показано, что $\underline{C}(1)=(\sqrt{10}+3) /(6 \sqrt{2 \pi})$.

Чтобы конкретизировать порядок малости величины $o\left(L_{n}^{2+\delta}\right)$, фигурирующей в следствиях 3 и 4 , для начала заметим, что из следствия 2, очевидно, вытекает следуюшее утверждение.

Следствие 5. В условиях (1) u (2) $c \delta \in(0,1)$ для любого $\varepsilon>0$ существует число $c(\delta, \varepsilon) \in(0, \infty)$ такое, что выполнено неравенство

$$
\rho\left(F_{n}, \Phi\right) \leqslant(C(\delta)+\varepsilon) L_{n}^{2+\delta}+c(\delta, \varepsilon)\left(L_{n}^{2+\delta}\right)^{1+(1-\delta) / \delta} .
$$

Комбинируя утверждения следствий 4 и 5 , мы получаем следуюший результат.

Следствие 6. В условиях (1) u (2) с $\delta \in(0,1)$ существует число $c(\delta) \in(0, \infty)$ такое, что выполнено неравенство

$$
\rho\left(F_{n}, \Phi\right) \leqslant \frac{1}{\pi} L_{n}^{2+\delta}+c(\delta)\left(L_{n}^{2+\delta}\right)^{1+(1-\delta) / \delta} .
$$

От присутствия добавки $\varepsilon$ в абсолютной константе при первом слагаемом в правой части (16) можно избавиться совсем. Однако платой за это будет некоторое ухудшение скорости убывания второго слагаемого. Чтобы в этом убедиться, заметим, что с помощью элементарных рассуждений можно получить оценки

$$
\gamma(\omega) \leqslant 2 \pi+\frac{4}{\pi \omega}
$$

$$
d(\delta, \varepsilon) \geqslant M(\delta) \varepsilon^{2 / \delta},
$$


где

$$
\begin{aligned}
M(\delta)= & \{8 \pi(1+\delta)(2+\delta)\}^{2 / \delta}\left\{2^{1-\delta / 2}(1+\delta)(2+\delta) \Gamma\left(\frac{2+\delta}{2}\right)+\left(6+\delta+\frac{2^{1-\delta}}{1+\delta}\right)\right. \\
& \left.\times\left[2^{1-\delta / 2} \Gamma\left(\frac{2+\delta}{2}\right)+\pi(1+\delta)(2+\delta)\right]\right\}^{-2 / \delta} .
\end{aligned}
$$

Подставляя оценки (17) и (18) в неравенство (15), мы получим

$$
\begin{aligned}
\rho\left(F_{n}, \Phi\right) \leqslant & C(\delta) \frac{\beta^{2+\delta}}{n^{\delta / 2}}+(\varepsilon+\omega C(\delta)+\varepsilon \omega) \frac{\beta^{2+\delta}}{n^{\delta / 2}} \\
& +\frac{\sqrt{2}}{\sqrt{\pi} M(\delta) \varepsilon^{2 / \delta}}\left(\pi+\frac{1}{\pi}+\frac{\omega \pi}{2}+\frac{2}{\pi \omega}\right) \frac{\beta^{2+\delta}}{\sqrt{n}} .
\end{aligned}
$$

Полагая теперь в последнем неравенстве $\omega=n^{-a}$ и $\varepsilon=n^{-b}$ с $a>0$ и $b>0$, можно заметить, что при

$$
a=b=\frac{\delta(1-\delta)}{4(1+\delta)}
$$

минимальная скорость убывания выражений, зависящих от $a$ и $b$, с ростом $n$ является наибольшей. Таким образом, мы окончательно приходим к следующему «разложению» оценки коэффициента $C_{n}(\delta)$ в теореме 1.

Следствие 7. В условиях (1) $u(2)$ при $\delta \in(0,1]$

$$
\rho\left(F_{n}, \Phi\right) \leqslant \frac{\beta^{2+\delta}}{n^{\delta / 2}}\left(C(\delta)+Q_{1}(\delta) \tau_{n}+Q_{2}(\delta) \tau_{n}^{2}+Q_{3}(\delta) \tau_{n}^{3}\right)
$$

$2 \partial e$

$$
\begin{gathered}
\tau_{n}=\tau_{n}(\delta)=n^{-(1-\delta) /(2(1+\delta))}, \\
Q_{1}(\delta)=1+C(\delta)+\frac{2 \sqrt{2}}{\pi^{3 / 2} M(\delta)}, \quad Q_{2}(\delta)=1+\frac{\sqrt{2}\left(1+\pi^{2}\right)}{\pi^{3 / 2} M(\delta)}, \quad Q_{3}(\delta)=\frac{\sqrt{\pi}}{\sqrt{2} M(\delta)} .
\end{gathered}
$$

$\mathrm{K}$ примеру, если $\delta=\frac{1}{2}$, то имеет место неравенство

$$
\rho\left(F_{n}, \Phi\right) \leqslant \frac{\beta^{2+1 / 2}}{n^{1 / 4}}\left(0.1294+\frac{9.98}{n^{1 / 24}}+\frac{49.1008}{n^{1 / 12}}+\frac{21.8378}{n^{1 / 8}}\right) .
$$

Если же $\delta=\frac{3}{4}$, то

$$
\rho\left(F_{n}, \Phi\right) \leqslant \frac{\beta^{2+3 / 4}}{n^{3 / 8}}\left(0.0907+\frac{3.2435}{n^{1 / 14}}+\frac{12.6510}{n^{1 / 7}}+\frac{5.2896}{n^{3 / 14}}\right) .
$$

3 а м е ч а н и е 2 . С учетом неравенства $C(\delta) \leqslant 1 / \pi$ из следствия 7 мы получаем оценку

$$
\rho\left(F_{n}, \Phi\right) \leqslant \frac{\beta^{2+\delta}}{n^{\delta / 2}}\left(\frac{1}{\pi}+Q_{1}(\delta) \tau_{n}+Q_{2}(\delta) \tau_{n}^{2}+Q_{3}(\delta) \tau_{n}^{3}\right)
$$

из которой вытекает, что при малых $\delta$ константу в оценке скорости сходимости в центральной предельной теореме «портят» добавки, соответствующие более высоким степеням $1 / n$, нежели $\delta / 2$.

Так как $\beta^{2+\delta} \geqslant 1$, то из следствия 7 вытекает оценка

$$
\begin{aligned}
\rho\left(F_{n}, \Phi\right) \leqslant & C(\delta) L_{n}^{2+\delta}+Q_{1}(\delta)\left(L_{n}^{2+\delta}\right)^{1+(1-\delta) /(2(1+\delta))} \\
& +Q_{2}(\delta)\left(L_{n}^{2+\delta}\right)^{1+(1-\delta) /(1+\delta)}+Q_{3}(\delta)\left(L_{n}^{2+\delta}\right)^{1+3(1-\delta) /(2(1+\delta))}
\end{aligned}
$$

В правой части этой оценки порядки слагаемых, очевидно, упорядочены по возрастанию. Поэтому справедливо следуюшее утверждение.

Следствие 8. В условиях (1) $u(2)$ при $\delta \in(0,1] u n \geqslant \beta^{2+\delta}$ имеет место оченка

$$
\rho\left(F_{n}, \Phi\right) \leqslant C(\delta) L_{n}^{2+\delta}+Q(\delta)\left(L_{n}^{2+\delta}\right)^{1+(1-\delta) /(2(1+\delta))}
$$

где $C(\delta)$ определено соотношением (11),

$$
Q(\delta)=Q_{1}(\delta)+Q_{2}(\delta)+Q_{3}(\delta),
$$

а коэффициенты $Q_{j}(\delta), j=1,2,3$, определень в следствии 7. 
4. Доказательство теоремы 1. Из леммы 2 вытекает, что

$$
\rho\left(F_{n}, \Phi\right) \leqslant \frac{1}{2 \alpha(\gamma)-1}\left[\frac{1}{2 \pi} \int_{-\gamma / v}^{\gamma / v}\left|\frac{f_{n}(t)-e^{-t^{2} / 2}}{t}\right| d t+\frac{v \alpha(\gamma)}{\sqrt{2 \pi}}\right] \equiv \frac{I_{1}+I_{2}}{2 \alpha(\gamma)-1}
$$

Величину $I_{1}$ оценим с помощью леммы 1 и положим

$$
v=v_{n}=\frac{\gamma \beta^{2+\delta}}{d \sqrt{n}}, \quad d \in(0, \sqrt{2})
$$

Получим

$$
\begin{aligned}
I_{1} & \leqslant \int_{|t| \leqslant d \sqrt{n} / \beta^{2+\delta}}\left|\frac{f_{n}(t)-e^{-t^{2} / 2}}{t}\right| d t \leqslant \frac{a_{2}(d)}{2 \pi} \frac{\beta^{2+\delta}}{n^{\delta / 2}} \int_{-\infty}^{+\infty}|t|^{1+\delta} \exp \left\{-b_{1}(d) t^{2}\right\} d t \\
& =\frac{a_{2}(d)}{\pi} \frac{\beta^{2+\delta}}{n^{\delta / 2}} \int_{0}^{+\infty} t^{1+\delta} \exp \left\{-b_{1}(d) t^{2}\right\} d t+\frac{a_{2}(d)}{2 \pi\left[b_{1}(d)\right]^{1+\delta / 2}} \frac{\beta^{2+\delta}}{n^{\delta / 2}} \int_{0}^{+\infty} y^{\delta / 2} e^{-y} d y \\
& =\frac{\Gamma((2+\delta) / 2) a_{2}(d)}{2 \pi\left[b_{1}(d)\right]^{1+\delta / 2}} \frac{\beta^{2+\delta}}{n^{\delta / 2}} \equiv K_{\delta}(d) \frac{\beta^{2+\delta}}{n^{\delta / 2}}
\end{aligned}
$$

При этом область возможных значений параметра $d$ сужается до интервала $\left(0, d_{0}(\delta)\right)$, определяющего область сходимости интеграла в $(19)$, где $d_{0}(\delta)$ - единственный нуль монотонно убывающей функции $b_{1}(d)$ на $(0, \sqrt{2})$.

Подставляя указанное выше значение $v=v_{n}$ в выражение для $I_{2}$, получаем

$$
I_{2}=\frac{v \alpha(\gamma)}{\sqrt{2 \pi}}=\frac{\gamma \alpha(\gamma)}{\sqrt{2 \pi} d} \frac{\beta^{2+\delta}}{\sqrt{n}}
$$

Теорема доказана.

\section{СПИСОК ЛИТЕРАТУРЫ}

1. Бхаттачария P. Н., Ранга Рао Р. Аппроксимация нормальным распределением. М.: Наука, 1982, 286 с.

2. Золотарев B. М. Абсолютная оценка остаточного члена в центральной предельной теореме. - Теория вероятн. и ее примен., 1966, т. 11, в. 1, с. 108-119.

3. Золотарев $B$. M. Современная теория суммирования независимых случайных величин. М.: Наука, 1986, 415 с.

4. Королев В. Ю., Шевчова И. Г. О точности нормальной аппроксимации. I. - Теория вероятн. и ее примен., 2005, т. 50, в. 3, с. 353-366.

5. Лоэв М. Теория вероятностей. М.: ИЛ, 1962, 719 с.

6. Мацкявичюс B. K. О нижней оценке скорости сходимости в центральной предельной теореме. - Теория вероятн. и ее примен., 1983, т. 28, в. 3, с. 565-569.

7. Осипов Л. В. У точнение теоремы Линдеберга. - Теория вероятн. и ее примен., 1966, т. 11, в. 2, с. 339-342.

8. Петров В.В. Суммы независимых случайных величин. М.: Наука, 1972, 416 с.

9. Феллер B. Введение в теорию вероятностей и ее приложения. Т. 2. М.: Мир, 1984, $752 \mathrm{c}$.

10. Чистяков Г. П. Новое асимптотическое разложение и асимптотически наилучшие постоянные в теореме Ляпунова. I. - Теория вероятн. и ее примен., 2001, т. 46, B. 2 , с. $326-344$.

11. Шиганов И. С. Об уточнении верхней константы в остаточном члене центральной предельной теоремы. - Проблемы устойчивости стохастических моделей. Труды ВНИИСИ, 1982, с. 109-115.

12. Bentkus $V$. On the asymptotical behavior of the constant in the Berry-Esseen inequality. - J. Theoret. Probab., 1994, v. 7, № 2, p. 211-224.

13. Esseen C.-G. A moment inequality with an application to the central limit theorem. Skand. Aktuarietidskr., 1956, v. 39, p. 160-170. 
14. Paditz L. Über eine globale Fehlerabschätzung im zentralen Grenzwertsatz. - Wiss. Z. Hochschule für Verkehrswesen «Friedrich List» Dresden, 1986, v. 33, № 2, p. 399-404.

15. Paditz $L$. On the error-bound in the nonuniform version of Esseen's inequality in the $L_{p}$-metric. - Statistics, 1996, v. 27, №3-4, p. 379-394.

16. Tysiak $W$. Gleichmäßige und nicht-gleichmäßige Berry-Esseen-Abschätzungen. Dissertation. Wuppertal, 1983.

Поступила в редакцию 22.IV.2005

(c) 2005 г.

ЛЕБЕДЕВ А. В.*

\section{МАКСИМАЛЬНЫЕ ВЕТВЯЩИЕСЯ ПРОЦЕССЫ С НЕОТРИЦАТЕЛЬНЫМИ ЗНАЧЕНИЯМИ ${ }^{1)}$}

Проводится обобщение максимальных ветвящихся процессов (введенных Дж. Ламперти) с области значений $\mathbf{Z}_{+}$на $\mathbf{R}_{+}$. Изучены некоторые их свойства, доказана эргодическая теорема, приведены примеры. Указаны приложения процессов в теории массового обслуживания.

Ключевые слова и фразы: максимальные ветвящиеся процессы, эргоцические теоремы, ассоциированность, монотонность по параметрам, экстремумы, вентильные бесконечнолинейные системы.

1. Введение. Параллели между теорией суммирования и теорией экстремумов случайных величин давно и хорошо известны.

Так, центральной предельной теореме (и ее аналогам) соответствует теорема об экстремальных типах, устойчивым распределениям - максимум-устойчивые $[1$, гл. 1], устойчивым процессам Леви - экстремальные процессы $[2, \S 6.5]$. При отказе от независимости случайных величин в обоих случаях вводятся некоторые условия перемешивания, позволяющие получать предельные теоремы и в ситуации зависимости.

Классическими объектами исследования в теории случайных процессов являются ветвящиеся процессы Гальтона-Ватсона (с одним типом частиц и дискретным временем) [3], [4]. Оказывается, для них также возможно разумным образом построить «экстремальные» аналоги, которые и называются максимальными ветвящимися процессами (МВП).

А именно, рассматриваются случайные процессы со значениями в $\mathbf{Z}_{+}$, заданные стохастически рекуррентными формулами вида

$$
Z_{n+1}=\bigvee_{m=1}^{Z_{n}} \xi_{m, n}
$$

где через $\bigvee$ обозначена операция взятия максимума и $\xi_{m, n}, m \geqslant 1, n \geqslant 0,-$ независимые случайные величины с общим распределением $F$ на $\mathbf{Z}_{+}$. Полагаем (как и в случае суммирования), что результат взятия максимума «нуль раз» (при $Z_{n}=0$ ) равен нулю.

* Московский государственный университет им. М.В. Ломоносова, механикоматематический факультет, кафедра теории вероятностей, Ленинские горы, 119992 Москва, Россия; e-mail: alebedev@mech.math.msu.su

1) Работа выполнена при поддержке грантов РФФИ 00-01-00131, 03-01-00724, 04-01-00700 и НШ 1758.2003.1. 